\title{
Métamorphose et Rationalité Mythique Chez Lygia Clark
}

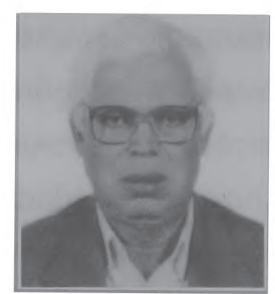




\section{Résumé}

En admettant, avec Cassirer, que le mythos est bien le volet du langage où "non seulement le mot garde son pouvoir figurateur originel, mais en plus il le renouvelle, par sa transformation en expression artistique", je me propose de rechercher les primitives figuratives de ce qu'on a coutume d'appeler, depuis l'exposition tenue en 1967 au Musée d'Art Moderne de Rio de Janeiro, la nouvelle objectivité brésilienne.Ce qui, dans la dite objectivité, retient mon attention, ce sont les métamorphoses spatiales mouvantes et incessantes mises en avant par Lygia Clark. Partant de l'affirmation de Suely Rolnik, que "Lygia épure les objets jusqu'à un presque-rien", je vois les métamorphoses clarkiennes comme des exploitations systématiques des atavismes morphiques du corps, soit des virtualités figúrales à l'oeuvre dès l'intérieur de l'objet - en l'occurrence, le corps propre de l'observateur.

Mots clés

virtualité figúrales, métamorphose, corps

\section{Resumo}

Admitindo, corn Emst Cassirer, que o mythos é a vertente da linguagem em que "a palavra não apenas conserva seu poder figurador original, mas o renova, ao se transformarem expressão artística", proponho-me à busca dos primitivos figurativos do que se convencionou chamar, a partir da exposição realizada em 1967 no Museu de Arte Moderna do Rio de Janeiro, nova objetividade brasileira. O que, nessa objetividade, retém minha atenção são as metamorfoses espaciais instáveis e incessantes propostas por Lygia Clark; vejo-as como explorações sistemáticas dos atavismos módicos do corpo, ou seja, como virtualidades figurais que atuam desde o interior do objeto, no caso, o corpo próprio do observador.

\section{Palavras-chave}

virtualidades figurais, metamorfose, corpo. 


\begin{abstract}
I e temps' est désormais révolu où la Sémiotique avait pour soucis _majeurs les systèmes de signes et leur stabilité structurelle. Cette Sémiotique privilégiait des procédures telles que la catégorisation, l'identification d'unités, ainsi que la mise en évidence de différences significatives; elle avait pour but les descriptions, les classifications, bref, il s'agissait d'une Sémiotique à dominante taxinomique. Lorsqu'elle attaquait l'explication, elle le faisait selon le versant duXoyoÇ, où, d'après Ernst Cassirer, "les mot́s se ramènent à de simples signes conceptuels" (1972, p. 105). S'inspirant à présent de l'affirmation de Louis Hjelmslev selon laquelle on ne peut rien faire de bon dans les sciences du langage sans dépasser le signe, la Sémiotique s'ouvre vers l'infra-signique pour y contempler un autre versant aussi radical et important du langage, celui du (JA)0oÇ, en vue d'y définir les stratégies par lesquelles "non seulement le mot garde son pouvoirfigurateur originel, mais en plus il le renouvelle, lorsqu'il se transforme en expression artistique"

Parmi les publications portant sur le mouvement néo-concrétiste, j'aimerais attirer l'attention sur $A$ invenção de Hélio Oiticica, de C. F Favaretto, "Lygia Clark: uma experiência radical", de F Gullar, Obra-
\end{abstract}

* Este texto é uma transcrição de palestra proferida no VI Congresso da Associação Internacional de Semiótica Visual (AISV), realizado em Siena (Itália) em junho de 1998. 
trajeto, de M. A. Milliet, Lygia por Lygia, de M. Duarte (org.), et enfin "Um singular estado de arte", de S. Rolnyk; ce sont là les principaux inspirateurs de l'inflexion de ma recherche vers ce qú on a convenu d'appeler, depuis l'exposition tenue en 1967 au Musée d'Art Moderne de Rio de Janeiro, la nouvelle objectivité brésilienne.

$A$ l'instar de ce que j'avais développé dans Figurativizaçâo $e$ metamorfose. O mito de Norciso (1995), où j'ai abordé, d'un côté, la métamorphose dédensifiante de Constantin Brancusi, et, d'un autre côté, les métamorphoses exacerbées d'Henri Michaux, ce qui $\mathrm{m}$ 'a d'abord frappé dans cette objectivité c'est, d'un côté, la gestualité patiente, dédensifiante de la production d'Anna Maria Maiolino, toujours attentive à la volonté plastique de l'objet - expression par laquelle Ugo Boccioni (1975, p. 102) désigne la potentialité plastique de la matérialité même de l'objet -, et, de l'autre côté, les métamorphoses spatiales agitées, incessantes, proposées par Lygia Clark. ${ }^{1}$

Pour scander l'entrée sémiotique dans l'univers clarkien, je me laisse guider par les ritournelles utilisées par S. Rolnyk (1994) pour parler de Baba antropofâgica (Bave anthropophagique), datant de 1973 :

- mémoire du corps,

- corps flux-bave,

- dé-réification de la subjectivité du spectateur,

- épuration de l'objet jusqu'à un presque-rien.

La mémoire du corps (laquelle "ne vise pas à chercher une forme ni dans le passé, ni dans le futur, mais l'expérience de vivre le participe présent de l'évolution incessante des formes" - c'est moi qui souligne)

1. “[...] nous ne composons pas dans une surface, mais celle-ci est utilisée pour exprimer un temps intérieur de l'espace même qu'elle exprime. Il y a donc une dynamique constante: l'espace se fait, se défait et se refait en permanence" (CLARK, 'Trajetória até o movimento neoconcreto", apuei Duarte, 1995).

Significação $13 \cdot 130$ 
intéresse la compréhension sémiotique de la métamorphose eu égard au privilège accordé par Rolnyk à ladite évolution et à l'acte de la vivre dans l'ici et maintenant.

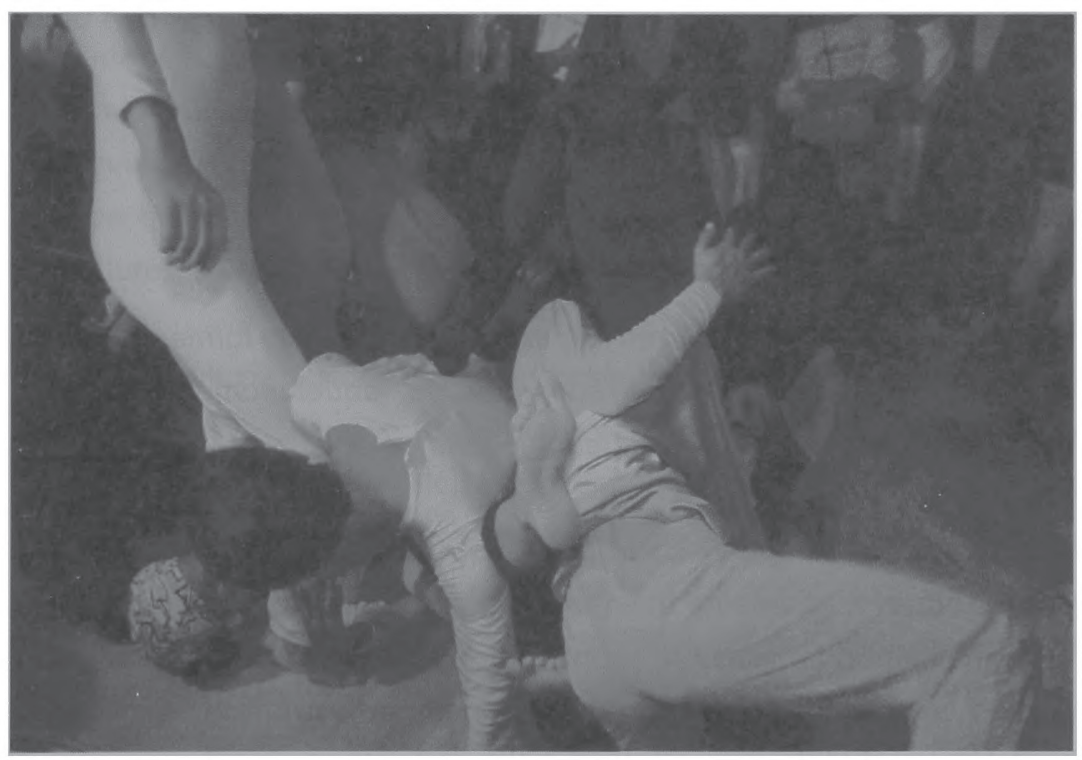

Nostalgia do Corpo - Corpo Coletivo, 1965-1988 - Foto Sérgio Zalis.

Le corps flux-bave se conçoit (sous l'inspiration du corps sans organes d'Artaud-Deleuze-Guattari ${ }^{2}$ ) comme "une agitation bouillante de flux de salive, de lignes, de bouches, de mains, dans des mouvements d'attraction et de répulsion, qui produisent des constellations"

$\mathrm{Ce}$ que je rebaptise comme dé-réification de la subjectivité du spectateur, c'est la principale stratégie discursive pour atteindre le singulier état d'art sans art: "En secouant la position individuelle et collective du spectateur, Lygia veut la dé-réifier, de façon à l'amener à se laisser découdre et coudre par le bouillonnement du travail souterrain des forces et des flux de notre Bicho (bête) [...]"

2. Cf. Mauro Sá R. Costa, O corpo sem órgãos e o sentido como acontecimento, in: Assis SILVA, 1996, p. 97-107. 
Subsumées par la remarque générale sur la subjectivation, ces ritournelles me conduisent à l'hypothèse que les métamorphoses clarkiennes seraient l'exploitation systématique d'atavismes morphiques, c'est-à-dire des virtualités figuratives agissant depuis l'intérieur de l'objet lui-même:

un presque-rien qui reste et qui est l'essentiel de ce qui dans l'objet mobilise... ce qui opère, dans le corps, l'expérience de la déstabilisation de la subjectivité du spectateur, tout en lui permettant de vivre la forme au moment même de son naufrage, lequel moment est aussi celui d'une germination.

Envisagés sous l'angle de la Sémiotique post- De l'imperfection, ces propos nous font admettre que l'expérience clarkienne, dans le trajet de l'Art vers l'état d'art sans Art, problématise des aspects de la production discursive touchant de près aux questionnements suivants:

- plutôt que de faire ressortir la stabilité de la structure qui engendre et soutient les termes discrets (visée discrétisante), cette Sémiotique se soucie de mettre en lumière non seulement les processus qui mènent à la stabilité structurelle, mais aussi ceux qui la minent (FONTANILLE, 1992, p. 12). Miner la stabilité structurelle, c'est bien ce que visent Hélio Oiticica et Lygia Clark, les principaux proposants de la nouvelle objectivité brésilienne. Lorsque Lygia veut amener son spectateur à (re)-éprouver l'expérience de la déstabilisation de la subjectivité, elle met en oeuvre une façon d'énoncer bien différente de la conception d'énonciation en tant qu'appropriation individuelle d'un système sémiotique: elle propose une praxis énonciative qui sélectionne, actualise, convoque plus d'un système sémiotique et qui, tout en les travaillant, les constitue en même temps qu'elle s'en nourrit (FONTANILLE, 1992, p. 11). Conduisant le spectateur à "se laisser découdre par le

Significaçâo $13 \cdot 132$ 
bouillonnement du travail souterrain des forces de notre Bicho (bête)", Lygia remplace le rôle de l'artiste qui produit pour la jouissance et la consommation d'autrui par celui de production-en-groupe stimulée et accueillie par le "propositor" (proposant). ${ }^{3} \mathrm{~A}$ en croire Fontanille, nous aurions là une praxis énonciative extrêmement sensible à l'analyse sémiotique, dans la mesure où elle déstabilise le système sémiotique et son usage, défaisant les découpages propres à telle ou telle culture, si bien qu'elle en dessine de nouveaux pour finalement réinventer les cultures (Fontanille, 1992, p. 11 , adaptation). ${ }^{4}$

La capacité de déstabiliser, de déconstruire, de défaire des découpages, rend la praxis énonciative (aussi bien celle que propose Lygia que celle conceptualisée par Fontanille), sinon identique, du moins très voisine des conceptions de métamorphose que j'ai avancées à l'occasion de ma lecture du mythe de Narcisse d'après Ovide, J. Lacan/A. Vallejo, Brancusi, Henri Michaux et Salvador Dali (ASSIS SILVA, 1995), développant les concepts suivants:

3. “Nous sommes les 'propositores': nous portons en nous un grand vide. Nous vous

proposons de donner du sens à ce même vide. Ce n'est pas que nous n'ayons pas le courage de le formuler, mais nous ne trouvons plus de sens dans une proposition complète.

Nous sommes les 'propositores': nous demandons votre aide. Faites usage de ce vide, et complétez-le, car nous nous en trouverons pleins de sens. Nous sommes le moule de la vie. Il vous appartient de souffler dans notre moule le sens de notre existence. Nous sommes les "propositores': nous enterrons l'œuvre d'art en tant que telle, et nous vous demandons la parole, pour que la pensée survive au moyen de l'acte.

Nous sommes les 'propositores': nous ne sommes pas allés le chercher dans le passé; nous ne vous proposons pas non plus de rencontre dans le futur, mais il s'agit du maintenant.

Nous sommes les 'propositores': thermomètre n'affichant pas la température, car celleci est en vous" (CLARK, Neoconcretismo, irr. DUARTE, 1995).

4. Les Manifestações ambientais reproposent la "réalisation créative" tout en y intégrant le collectif par le redimensionnement culturel des protagonistes (artiste et participants) (Favaretto, 1992, p. 121). 
- la renaissance incessante, ainsi que l'idée de production issue de la destruction: la métamorphose implique, d'une façon générale, la destruction, ou plutôt la déconstruction d'une forme antérieure qui ne disparaît pas complètement (p. 31);

- la métamorphose radicale en tant que parole du monde, en tant que transformation d'un état de choses dans un état de signe (p. 64);

- le support figurai profond, jouant le rôle d'une sorte de sténogramme à partir duquel sera générée la nouvelle forme. Ce support fonctionne à la manière d'une matrice figurale ${ }^{5}$ (p. 94);

- le passage d'un état à un autre contraint par les possibilités constructives de l'objet lui-même: celui-ci porte en lui-même ses possibilités de transformation (p. 180);

- la métamorphose en tant que circulation infinie: chez le dernier Michaux, le sujet, comme un Narcisse-Protée, devient un monde ouvert jusqu'à la mort; la diversité et la multiplicité y régnent souveraines (p. 217 );

- last but not least, l'exploitation systématique des atavismes morphiques dont cette stratégie recommande de retenir la vigueur figurative et de la lire à la lumière des possibilités constructives internes de l'objet: il existe à l'intérieur de chaque forme des forces ataviques (= renvoyant à un étymon archaïque ${ }^{6}$ ) lesquelles soutiennent, depuis l'instance profonde, l'engendrement de la forme; chez Dali, l'exploitation de ces atavismes est une éclosion libidineuse, carnivore et en même temps divine (dionysiaque), en pleine conformité donc avec l'oralité cosmique,

5. Concept apparenté, bien que non identifiable, au figurai lyotardien: registre primitif récurrent, matrice cellulaire où s'inscrit le trauma des premiers rapports de l'être à l'environnement. Pour moi, le figurai consiste en un dépouillement de la figure hjelmslevienne.

6. Pour illustrer cela, je renvoie à Henri Michaux, Postface à plume-. "Le jeune puma naît tacheté. Ensuite, il surmonte les tachetures. C'est la force du puma contre l'ancêtre, mais il ne surmonte pas son goût de carnivore, son plaisir à jouer, sa cruauté". Ou bien à Lemoine-Luccioni: "Des mots anciennement reçus comme des choses à un âge sans langage codifié. Ils sont amalgamés aux pulsions les plus primaires et en restent indépassables" \{Ij: rêve du cosmonaute, cité par WII,LF.MART, in Assis SII.VA, 1996, p. 160).

Significaçâo $13 \quad 134$ 
mortelle et cannibale de sa profession esthétique: la belleza será comestible o no será (p. 235).

Le poids de la mémoire du corps chez L. Clark demande à être examiné dans l'optique de l'ouverture vers la corporalité, posée par le Greimas de De l'imperfection comme un élément constitutif du sujet du discours.

Sur l'axe corps-langage, cette ouverture est pensable en termes de médiation entre la macrosémiotique de la Langue Naturelle et celle du Monde Naturel. Y tiennent une place centrale les concepts de figure et de figurativité. Une fois rompue, la figurativité - qui "n'est pas une simple ornementation des choses, elle est cet écran du paraître dont la vertu consiste à entr'ouvrir, à laisser entrevoir, grâce ou à cause de son imperfection, comme une possibilité d'outre-sens" (GREIMAS, 1987, p. 78) - pointe vers un sensible immanent, au statut mythique, sous-jacent ou glissant sous les exfoliations du discours; mythique car il s'y produit la transformation fondamentale de la relation Sujet-Objet, événement unique, extraordinaire, établissement instantané d'un "état de choses" Ce que Greimas y aperçoit, ce n'est pas la morphologie modale, ni la sémantique passionnelle, même pas la sémantique thématique, mais le statut figurái de la métamorphose instantanée de l'état de choses. La pensée sémiotique rejoint ici l'intuition du plasticien. Pour Boccioni, "la réalité n'est pas l'objet, mais la transfiguration que celui-ci subit lorsqu'il s'identifie au sujet" (1975, p. 69) (c'est moi qui souligne). J'ai déjà fait allusion à A. M. Maiolino, qui dit: "Moi et elles (les lignes), nous nous entendons bien dans l'intimité du faire" - révélant ainsi non seulement l'obéissance de la matière à ses pulsions d'artiste, mais aussi une sorte de fusion entre sa volonté et les impositions du matériau choisi. ${ }^{7}$ Pour Lygia Clark, l'œuvre est un non-

7. “Dans les simples gestes manuels [il se produit] la saisie première de la matière et sa transformation significative. Il en sourd un concept premier en forme de madère, un concept-argile, concret, tactile... une forme de langage, un objet-langage" (VENÂNCIO FILHO, 1995, adaptadon). 
objet, c'est-à-dire, non pas une représentation, mais une présentation dont la signification s'enrichit de la participation du spectateur, stimulée par l'objet lui-même... Plutôt que contemplé, celui-ci est mis en action par le spectateur qui, tout en libérant les potentialités contenues dans l'objet même, est du même coup libéré par celles-ci (Cf. MILLIET, 1 992, p. 85$).^{8}$

On pourrait ajouter à ces convergences que Lygia aussi bien que Hélio opposent à ce qu'ils appellent réductionnisme rationaliste dans le Concrétisme, ce que Merleau-Ponty nomme "sujet voué au monde":

Le monde n'est pas un objet dont je possède par devers moi la loi de constitution, il est le milieu naturel et le champ de toutes mes pensées et de toutes mes perceptions explicites. La vérité n'habite pas seulement l'homme intérieur, ou plutôt il n'y a pas d'homme intérieur, l'homme est au monde, c'est dans le monde qu'il se connaît (apud MILUET, ibidem,

p. 26).

Curieuse et suggestive convergence à rebours: Lygia et Hélio in-corporant à l'œuvre la signification corporelle, sous le patronage du même Merleau-Ponty dont la présence insémine les premiers chapitres de Sémantique structurale, écrit en 1964, et imprègne tout le petit-grand livre de Greimas paru en 1987, De l'imperfection.

Envisagée sous l'opposition AoyoÇ/juvOoçqui structure le langage, la

8. Aussi semble-t-il intéressant de rapprocher ces propos de ce que dit $\mathbf{H}$. Oiticica au sujet du Parangolê: "Ce n'est pas une chose à mettre sur le corps, à exhiber. L'expérience de celui qui s'habille pour quelqu'un qui le regarde du dehors, ou celle des gens qui mettent simultanément les choses, ce sont là des expériences simultanées, ce sont des multiexpériences. Il ne s'agit donc pas du corps en tant que support de l'œuvre; au contraire, c'est l'incorporation du corps dans l'œuvre et de l'œuvre dans le corps. J'appelle cela 'incorpo-ration"” (Oiticica, Aspiro aogrande labmnto, apud Favaretto, 1992, p. 107).

Significaçâo $13 \quad 136$ 
façon dont Lygia et Hélio opposent leur faire au Concrétisme appelle une relecture en termes de constitution d'une poétique où se syncrétisent le penchant pour le hoyoÇ de l'épistémè concrète et le penchant pour le |il)0oÇ de l'épistémè néoconcrète. Dans cette optique, les traits sous lesquels Milliet (1 992, p. 24-6) et Favaretto (1992, p. 39) décrivent une telle opposition peuvent et doivent être repris dans le cadre du projet et des recherches en cours sur la constitution et le fonctionnement des configurations semi-symboliques. LeXoyoÇ tout comme le fivGoÇ entrent dans la formation des poétiques concrète et néoconcrète.

La première met l'accent sur la dédensification, le dépouillement de l'objet, par la recherche de ses qualités plastiques; la seconde, quant à elle, insiste sur le remplissage participatif de l'objet, tout en privilégiant la mobilisation du "spectateur", de façon à le faire partager avec le proposant l'entreprise éthico-politico-esthétique de remplissage du vide de l'objet en y investissant des sens existentiels (nous-ici-maintenant) trempé dans le découpage que le Tropicalisme - et, en son sein, le Néoconcrétisme opère dans l'imaginaire brésilien. ${ }^{9}$

Pourquoi faire? Pour dévorer l'importation des "-ismes", en démontant l'usage élitiste et marchand de l'art. Clark, Oiticica, tout comme les participants à l'Exposition Rio/67 résistent farouchement à leur rangement comme "ingénieurs de l'oisiveté de l'avenir" par une société en décomposition.

9. 'Nous sommes les 'propositores': nous demandons votre aide. Faites usage de ce vide, et complétez-le, car nous nous en trouverons pleins de sens. Nous sommes le moule de la vie. Il vous appartient de souffler dans notre moule le sens de notre existence" (LYGIA CLARK). 
Schématiquement:
dés-incorpo-ration
dépouillement
contemplation
1
épistémè concrète
in-corpo-ration
épaississement
participation
1
épistémè néoconcrète

La production de Lygia Clark comme un ensemble apparaît comme un chantier privilégié pour l'étude, de la figurativité dans le rôle que lui assignent les recherches actuelles sur la figurativisation et le semi-

symbolisme: celui d'une matrice fondant l'imaginaire. J'insiste ici sur la figurativité profonde, conçue en tant qu'instance d'une métamorphose radicale (ou métaphore vraiment radicale selon le mot de Cassirer), où les percepts, figures du monde engendrées par la perception, se transmuent en sèmes. A cet égard, l'épuration de l'objet jusqu'à un presque-rien par Lygia Clark s'avère être un terrain intéressant pour la vérification et le questionnement de la quête d'une intersémiose fondamentale précédant toutes les pressions que fait peser le verbal sur le sujet de l'énonciation. Le moment est donc venu pour la Sémiotique de reprendre cette question à partir d'une objectivité immanente, d'une protoforme constitutive du sujet et de son intentionnalité, ou bien, comme le suggèrent Greimas et Fontanille (1991, p. 25), en termes d'une valence qui, comme une ombre, suscite le pressentiment de la valeur: la relation sujet-objet, si persistante dans les propositions clarkiennes, apparaît sous un jour spécial dès qu'on la conçoit à partir d'une proto-objectivité de nature figúrale laquelle attire le sujet avant même sa constitution modale, établissant par là le lien premier entre l'intelligible et le sensible. ${ }^{10}$

10. Nous en trouvons un bon exemple dans la petite main-Echo tenant un ouf minuscule au sommet de la montagne située à droite dans le tableau de Salvador Dalí, Metamorfosis de Narciso. Parole et image inextricablement mêlées dans un jeu-lutte de vie et de mort, 
En bref, les objets et les non-objets clarkiens intéressent la Sémiotique dans la mesure où ils pathémisent le sujet ou se font pathémiser par celui-ci, dans et par la médiation du corps. Si nous acceptons que non seulement nos "âmes", mais également nos corps se trouvent culturalisés à l'intérieur d'une culture (Greimas, 1991), et que la praxis énonciative déclenchée par Lygia opère la mise en place d'une nouvelle sensibilité et d'une nouvelle passionnalité, nous pouvons y voir une sorte de révolution: leur mise en texte est re-proposée non pas comme mise en forme mais comme mise en place exercée comme une mise en contexte, au sens jakobsonien. ${ }^{11}$

De telles considérations nous mènent à conclure que la recherche sur la métamorphose et la rationalité mythique chez Lygia Clark, et, par extension, dans la nouvelle objectivité brésilienne, peut avantageusement être conduite en tenant compte de la suggestion de Petitot:
La compréhension de l'articulation entre le registre pulsionnel hérité de la régulation animale et le registre d'idéaux faisant des pulsions non plus des "instincts" mais des opérateurs symboliques est sans doute la clef de i'élucidation des processus dialectiques de croyance, de passion, de manipulation et de

ayòv, mais qui, in extremis, lorsqu'ils atteignent la couche figurale où dort le mythe, se soutiennent sténogrammatiquement et réciproquement. A ce niveau-là, la voix pas plus que l'image ne peut mourir... Sujet et objet s'entrelacent dans la petite main, objet-lien, métaphore profonde, point de jonction/bascule des traits du Monde Naturel avec/ dans des traits de la Langue Naturelle (Assis Silva, 1995, p. 259).

11. L. Clark se met à partager avec les autres sa création, se déplaçant de l'espace institutionnel vers l'espace social; cela est affirmé avec autrement d'emphase par Oiticica: "A quoi bon cela servirait de jeter un 'pénétrable' dans un endroit quelconque, même sur une place publique, sans rechercher la moindre intégration et préparation à opposer à son sens unitaire? Il s'agit là d'un besoin profond et important, non seulement eu égard à l'origine de l'idée elle-même, mais en plus pour éviter que celle-ci ne s'égare dans des gratuités d'emplacement, de lieu, etc." (OITICICA, 22e Biennale Internationale de Sâo Paulo). 
séduction, bref, en définitive, des processus relevant

de la véridiction (1982, p. 32).

Véridiction, dire-vrai non-individuel dans un entre-deux, mais collectif ou plutôt communautaire. Discourant sur l'organisation sociale indoeuropéenne, Benveniste a pu dire que "chaque membre de (l'unité sociale) ne découvre son soi que dans l'entre-soi" (1969, v. 1, p. 321 ). Actualisant Benveniste par l'intermédiaire de Lucrèce pour qui (selon PARRET, 1996, p. 65) "l'être-au-monde et l'être-avec-les-autres sont un intercorps de besoins et de sensations dont le plaisir nous dit la vérité", nous sommes autorisés à conclure:

Le corps ne découvre son soi que dans l'entre-corps.

\section{Bibliographie}

ASSIS SILVA, I. 1995. Figurativizaçõo e metamorfose: o mito de Narciso. São Paulo: Edunesp. 1996. Corpo e sentido: a escuta do sensível. São Paulo: Edunesp.

BIENAL DE SÃo PAULO (FUNDAÇÃO) (s/d). 22a Bienal Internacional: Hélio Oiticica, Lygia Clark. Salas especiais. São Paulo: Fundação Bienal. BocCIONI, U. 1975. Dynamisme plastique. Lausanne: L'Age d'Homme. CASSIRER, E. 1972. Linguagem e mito. São Paulo: Perspectiva.

CIARK, L. s/d. Memória do corpo - o "dentro" e o "fora" Arquivo Lygia Clark. Rio de Janeiro: MAM.

DUARTE, M. 1995. Lygia por Lygia (versão preliminar). Rio de Janeiro: MAM.

Favaretto, C. F 1992. A invenção de Hélio Oiticica. São Paulo: Edusp. FonTANILLE, J. 1992. Les figures de l'instabilité. Projet d'Association au CNRS.

Significação $13 \quad 140$ 
GREIMAS, A. J. 1987 De l'imperfection. Périgueux: Pierre Fanlac. \& FONTANILLE, J. 1991. Sémiotique des passions. Paris: Seuil.

GULLAR, F 1959 Lygia Clark, uma experiência radical. Jornal do Brasil. Rio de Janeiro, 22 mar..

Merleau-Ponty, M. 1945. Phénoménologie de la perception. Paris:

Gallimard.

MILLIET, M. A. 1992. Lygia Clark. Obra-trajeto. São Paulo: Edusp.

Museu de Arte Moderna. 1967 Nova objetividade brasileira. Rio de Janeiro: MAM.

OITICICA, H. 1986. Aspiro ao grande labirinto (textos selecionados por L. Figueiredo, L. Pape e W. Salomão). Rio de Janeiro: Rocco.

PARRET, H. 1996. A verdade dos sentidos. Aula de semiótica lucreciana. In: ASSIS Silva. Corpo e sentido: a escuta do sentido. São Paulo: Edunesp, 1996, p. 45-65.

Petitot, J. 1982. Sur la décidabilité de la véridiction. Actes sémiotiques Documents, v. 4, n. 31, p. 21-40.

Rolnyk, S. 1994. Um singular estado de arte. Folha de S. Paulo. São Paulo, 4 dez.

VenânCIO FiLHO, P. 1 995. A mão que faz. Anna Maria Maiolino, Exposição itinerante (catálogo). 